08

\title{
Цезий с остаточным кислородом на цилиндрическом поликристалле вольфрама
}

\author{
() Б.М. Зыков, Т.М. Красненкова, Б.А. Лазба \\ Сухумский физико-технический институт АН Абхазии, \\ 384914 Сухум, Абхазия \\ e-mail: kras1946@mail.ru
}

Поступило в Редакцию 10 апреля 2019 г.

В окончательной редакции 20 мая 2019 г.

Принято к публикации 1 сентября 2019 г.

Методами низкоэнергетической пороговой электронной спектроскопии и контактной разности потенциалов определены химические состояния и изменение работы выхода неоднородной многоуровневой пленки во время адсорбции цезия при $295 \mathrm{~K}$ на цилиндрическом газофазном поликристаллическом вольфрамовом покрытии $(30 \mu \mathrm{m})$ сплава НбЦ-1 $(\mathrm{Nb}-1 \% \mathrm{Zr})$ с минимальной концентрацией остаточного кислорода. Определены химические состояния и изменение работы выхода этой пленки при последовательном прогреве до $1875 \mathrm{~K}$.

Ключевые слова: сплав ниобий-цирконий, вольфрамовое покрытие, остаточный кислород, адсорбция цезия, прогрев.

DOI: $10.21883 /$ JTF.2020.02.48822.160-19

\section{Введение}

В настоящее время наиболее эффективным для коллекторов термоэмиссионных преобразователей (ТЭП) тепловой энергии в электрическую, применяемых в космических ядерных энергетических установках, является газофазное поликристаллическое покрытие вольфрама $(\mathrm{W})(30 \mu \mathrm{m})$ на сплаве НбЦ-1 $(\mathrm{Nb}-1 \% \mathrm{Zr})$. При этом получаются наиболее стабильные и приемлемые по другим параметрам выходные характеристики. Однако до сих пор не ясно, существует ли возможность их дальнейшего улучшения. Это связано с трудностями в понимании химических процессов при наличии на поверхности такого коллектора как необходимых для его нормальной работы компонентов пленочной системы - кислорода $(\mathrm{O})$ и цезия $(\mathrm{Cs})$, так и других возможных примесей, в особенности наиболее вредных - углерода $(\mathrm{C})$, серы $(\mathrm{S})$ и их соединений с $\mathrm{O}$ и материалом подложки. Сложность этих процессов связана как с поликристалличностью покрытия и, следовательно, с его сильной дефектностью, так и с поливалентностью $\mathrm{W}$, приводящими даже при других фиксированных внешних условиях, например, потоке или концентрации $(n)$ адсорбатов и температуре $(T)$, к образованию на подложке одновременно нескольких типов химических соединений, в особенности при наличии других, кроме $\mathrm{O}$ и $\mathrm{Cs}$, примесей. При изменении внешних условий этот набор химических состояний также может изменяться. Поэтому изучение на фундаментальном уровне химических процессов для поликристаллической поверхности переходных металлов (Ме), применяемых для электродов ТЭП, представляет значительные трудности, в частности, отсутствие для поликристаллической поверхности в отличие от монокристаллической методов структурного анализа.
Известно, что структура является одним из важнейших атрибутов химического соединения. Поэтому исходя из актуальности для многих областей техники и технологии изучения химического состояния поликристаллической поверхности представляется рациональным применение для этих целей методов низкоэнергетической пороговой электронной спектроскопии (НЭПЭС) двух модификаций - низкоэнергетической спектроскопии упругорассеянных электронов (НЭСУРЭ) и низкоэнергетической пороговой спектроскопии истинно-вторичных электронов (НЭПСИВЭ). Они специально разработаны нами для определения энергетической электронной структуры валентной зоны (В3) твердых тел, т.е. по сути химического состояния их поверхности [1-5]. Эти методы дополнены некоторыми традиционными методами исследования поверхности, например, электронной оже-спектроскопией (ЭОС) для определения элементного и отчасти химического составов и контактной разности потенциалов (КРП) в варианте Андерсона для определения изменения работы выхода электронов $(\Delta \varphi)$. Для понимания процессов в сложных пленках с Cs необходимы знания как об исходных химических составах и состоянии поверхности, ее рельефе, так и о процессах, происходящих при ее взаимодействии с О. Результаты таких исследований для указанной подложки приведены в $[6,7]$.

\section{Методы исследования}

При изучении свойств сложных пленок c Cs применены: тот же образец, источник О, прибор, рабочие условия и методы исследования, что и в [7], с той же точностью измерений; источник ионов $\mathrm{Cs}^{+}$описан в [8]. 
Адсорбция Cs при различных по $T$ состояниях окисления во всех случаях производилась при $T$ подложки $295 \mathrm{~K}$ с шагом по $\Delta \varphi 0.1 \mathrm{eV}$. Во избежание частичной десорбции Cs из пленки от бомбардировки ее ионами $\mathrm{Cs}^{+}$из потока энергия (Е) последних варьировалась от $10 \mathrm{eV}$ в начале адсорбции до нуля в конце ее. Кроме того, для предотвращения влияния зондирующего пучка электронов на полученное при адсорбции Cs состояние пленки производилось вначале определение $\Delta \varphi$, а затем запись спектров. Таким образом, до прекращения адсорбции Cs и соответственно для изменений $\varphi$ для кривой адсорбции $(\Delta \varphi(t))$ получались в среднем около 20 спектров упругорассеянных электронов (СУРЭ). При монотонных изменениях в серии СУРЭ пороговые спектры истинно-вторичных электронов (ПСИВЭ) [1,2] для выявления в неоднородных пленках аморфных состояний в основном не записывались. Прогрев пленок с Cs начиная с $T=325 \mathrm{~K}$ производился в основном с шагом по $T(\Delta T)$, равном $50 \mathrm{~K}$, а в областях резких изменений состояний - с $\Delta T=25 \mathrm{~K}$. Он не прекращался и после полной десорбции Cs для того, чтобы выявить возможные стимулированные последним химические процессы. Таким образом, при прогреве каждой из пленок с Cs получали еще по 30-40 СУРЭ. Кроме того, при резких изменениях в СУРЭ затем записывались еще ПСИВЭ и ЭОС. Начальное состояние подложки получалось после прогрева при $1925 \pm 50 \mathrm{~K}$ предварительно максимально возможно очищенной поверхности $[6,7]$.

\section{Начальное состояние подложки}

Для начального состояния подложки в [6,7] показано, что прогрев образца при $T_{\max }=2075 \mathrm{~K}$ в вакууме $\sim 4 \cdot 10^{-8} \mathrm{~Pa}$ хотя и способствует удалению с W покрытия многих исходных примесей, но все же не приводит к получению атомно-чистой поверхности. На ней остаются $\mathrm{O}, \mathrm{Nb}$ и $\mathrm{C}$, последний по данным ЭОС в составе карбида $\mathrm{W}_{2} \mathrm{C}$ и монооксида $\mathrm{CO}$. Общепринятое для очистки от С циклическое окисление карбидов, согласно ЭОС, приводит к значительному уменьшению на поверхности их $n$ [6], но полностью они все же не удаляются. $\mathrm{CO}$, образующийся при окислении карбида остаточным кислородом, можно удалить повторным прогревом при $T=1375 \mathrm{~K}$. Однако прогрев при более высоких $T$ уже вызывает заметную диффузию С из объемных слоев материала образца к его поверхности, выделение его там (сегрегацию) и последующее окисление С или $\mathrm{W}_{2} \mathrm{C}$ остаточным $\mathrm{O}$ до $\mathrm{CO}$, часть которого сразу же десорбируется, а часть образуется вновь при остывании образца ниже $1375 \mathrm{~K}$ после выключения нагрева и остается на поверхности. Интегральное содержание $\mathrm{Nb}$ на поверхности после прогрева при $1975 \pm 100 \mathrm{~K}$ по данным НЭЭОС с $E$ первичного пучка электронов $\left(E_{p}\right)$, равной $220 \mathrm{eV}$, составляет $\sim 6.5 \mathrm{at} \%$ и вызывается его диффузией через $30 \mu \mathrm{m}$ покрытие $\mathrm{W}$. Но это не означает, что его $n$ в островковых пленках, в том числе и в виде соединений, не может быть выше. Корректное же определение $n$ остаточного $\mathrm{O}$ в настоящей работе затруднительно.

В $[6,7]$ установлено, что исследуемая подложка обладает достаточно дискретным трехуровневым потенциальным рельефом, который для образных представлений можно в достаточной мере считать геометрическим. Поэтому $\mathrm{Nb}$ расположен на гладких участках нижнего уровня в составе высокотермоустойчивого интерметаллического субоксида $\mathrm{Nb}_{2} \mathrm{~W}_{2} \mathrm{O}$ [7] типа $\mathrm{Nb}_{4} \mathrm{O}$ [9], аналогичного $\mathrm{Nb}_{2} \mathrm{Mo}_{2} \mathrm{O}$ [10]. Этим объясняется инертность W покрытия к высокотемпературному (ВТ) окислению [6,7]. СО расположен в основном на гладких участках среднего уровня, параллельных усредненной (базовой) поверхности, но менее протяженных, чем для $\mathrm{Nb}_{2} \mathrm{~W}_{2} \mathrm{O}$, например, на основаниях террас с наклонными стенками как линейных, так и винтовых в основном в составе субкарбонила $\mathrm{W}_{2} \mathrm{CO}$ с трехцентровой (мостиковой) связью $\mathrm{W}-\mathrm{C}-\mathrm{W}$ с подложкой и двойной $\mathrm{C}-\mathrm{O}$, а при наличии на этих участках плотноупакованных (ПУ) рядов атомов W (например, на гранях (110) или (112)) - и в составе субкарбонила WCO с сильной линейной донорно-акцепторной $\sigma$-связью $\mathrm{W}-\mathrm{C}$ и тройной $\mathrm{C}-\mathrm{O}$ в основном состоянии молекулы $\mathrm{CO}$ [11]. На верхнем уровне так же, как и в дефектах, свободных от $\mathrm{C}$, на других уровнях в зависимости от типа дефектов могут располагаться физадсорбированный на поляризационной связи остаточный $\mathrm{O}$ или молекулы $\mathrm{W}_{2} \mathrm{O}$ на мостиковой связи $\mathrm{W}-\mathrm{O}-\mathrm{W}$ с подложкой, последние в особенности в желобах террас или на их боковых стенках с достаточно малым наклоном к основаниям на среднем уровне или в бороздках от выхода на поверхность линейных (краевых) дислокаций, там, где невозможно образование молекул WCO или $\mathrm{W}_{2} \mathrm{CO}$, требующих вертикального расположения относительно базовой поверхности подложки. На нижнем уровне не исключено присутствие адсорбированного $\mathrm{O}$ на $\mathrm{Nb}_{2} \mathrm{~W}_{2} \mathrm{O}$ и в свободных от $\mathrm{C}$ или $\mathrm{W}_{2} \mathrm{C}$ объемных дефектах, из-за рельефа имеющих пониженную $E$ относительно более верхних уровней. Кислород попадает в эти дефекты, особенно при остывании образца после выключения нагрева. Карбид же $\mathrm{W}_{2} \mathrm{C}$ расположен на мостиковых связях $\mathrm{W}-\mathrm{C}-\mathrm{W}$ на стенках или между ними (в зависимости от ширины) в протяженных объемных дефектах, например, по границам зерен. Начальному состоянию подложки отвечает при $295 \mathrm{~K}$ отрицательное $\Delta \varphi=-0.25 \mathrm{eV}$, связанное с релаксацией (смещением в глубь подложки) верхних атомов $\mathrm{Nb}$ в $\mathrm{Nb}_{2} \mathrm{~W}_{2} \mathrm{O}$, вызываемое наличием в его молекулах под верхним слоем атомов подложки второй донорно-акцепторной связи $\mathrm{O}-\mathrm{Nb}$ [9] и, кроме того, с поглощением при остывании образца части остаточного кислорода с более высоких уровней в объемные дефекты, не занятые С или $\mathrm{W}_{2} \mathrm{C}$.

Актуальность настоящей работы вызвана необходимостью выяснить, требуется ли для получения оптимальных характеристик данного материала коллектора 
напуск в межэлектродный зазор ТЭП дополнительного кислорода, или же достаточно остаточного.

\section{Адсорбция цезия}

При адсорбции Cs из-за большого массива данных в настоящей работе описывается эволюция особенностей СУРЭ относительно начального состояния подложки, а на рисунке приводятся СУРЭ и ПСИВЭ лишь для начальных и конечных состояний пленочной системы или при появлении в процессах резких изменений. При попадании на подложку с минимальной $n$ остаточного $\mathrm{O}$, т.е. в ее начальном состоянии, уже первых порций Cs в отвечающих этому состоянию СУРЭ на рисунке (кривая 1,a) исчезает автоионизационный минимум (АИМ) при положительном напряжении $(U)$ $+0.2 \mathrm{~V}$ на анализаторе $E$ электронов типа тормозящего поля (АТП) [12]. Исходя из полярности $U$ для АИМ это говорит об экранировании чистых участков подложки, которые граничат с островками $\mathrm{Nb}_{2} \mathrm{~W}_{2} \mathrm{O}$ именно на нижнем уровне. АИМ при положительном $U$ на АТП связан с релаксацией части атомов подложки. При адсорбции Cs до $\Delta \varphi=-0.48 \mathrm{eV}$ интенсивность $(I)$ почти всех пиков в СУРЭ падает, за исключением пиков при 12.4 и $13.2 \mathrm{~V}$, соответствующих несвязывающим (заполненным) орбиталям О в СО и дифракции от подложки. Эти пики с ростом $I$ усредняются в прямоугольный, который теперь разумно приписать дублету от поляризованных подложкой $5 p$-орбиталей $\mathrm{Cs}^{+}$. По достижении $\Delta \varphi_{\min }=-1.63 \mathrm{eV}$ его $I$ возрастает в $\sim 2$ раза. С учетом $\varphi W=4.45 \mathrm{eV}$ и $\Delta \varphi$ начального состояния подложки $(-0.25 \mathrm{eV}) \varphi_{\min }$ при адсорбции Cs таким образом составляет $2.57 \mathrm{eV}$. Это более чем на $1.5 \mathrm{eV}$ превышает $\varphi_{\min }$ идеальных систем $\mathrm{Me}-\mathrm{O}-\mathrm{Cs}$ [8]. То есть на начальном этапе Cs адсорбируется статистически, в основном экранируя подложку. Уменьшение до $0.45 \mathrm{eV} E$ пика с начальной $E_{0}=0.55 \mathrm{eV}$, т.е. уменьшение поляризации кислородом поверхностных $d$-электронов подложки, не связанных в $\delta$-связях $\mathrm{Me}-\mathrm{Me}$, и рост $I$ пика при $2.3 \mathrm{~V}$ от химической связи О с подложкой в основном $\mathrm{W}-\mathrm{O}-\mathrm{W}$ говорит об одновременном уплотнении слабо связанного с подложкой О и образовании им дополнительных (вторичных) молекул $\mathrm{W}_{2} \mathrm{O}$. Об этом так же, как и о частичной очистке от О гладких участков верхнего уровня, говорит повторное появление при $\Delta \varphi=-0.97 \mathrm{eV}$ АИМ, но уже точно при $0 \mathrm{~V}$. Этим дополнительно подтверждаются факты перемещения О цезием на более нижние уровни и очистка верхних.

Вслед за этим начинается рост $I$ и $E$ пика при $0.35 \mathrm{~V}$. При $\Delta \varphi=-1.35 \mathrm{eV}$ появляется новый пик при $+0.15 \mathrm{~V}$, указывая на образование на верхнем уровне островковой пленки, не имеющей с подложкой химических связей. При этом $E$ пика при $0.35 \mathrm{~V}$ возрастает до $0.5 \mathrm{eV}$. При $\Delta \varphi=-1.43 \mathrm{eV}$ новый пик при $+0.15 \mathrm{~V}$ достигает $E=0$ при резком росте $I$ (рисунок, кривая 2,a). Это говорит о полном экранировании Ме

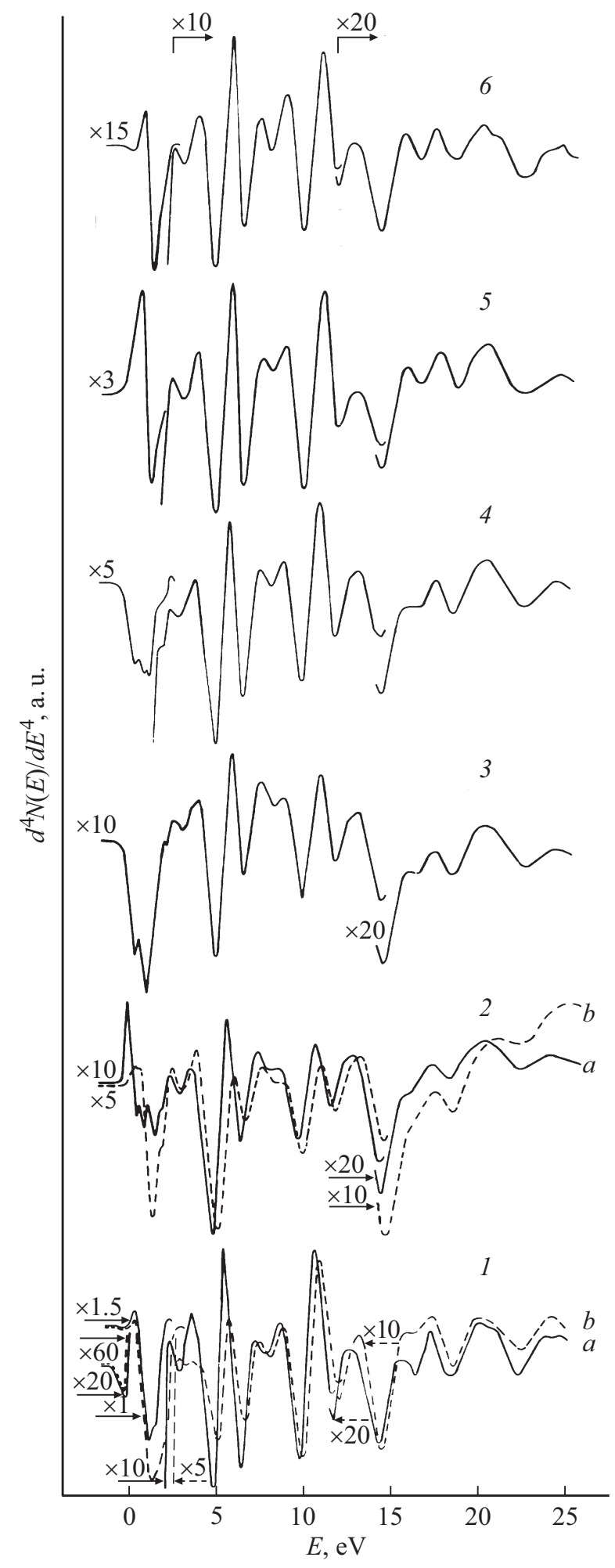

Пороговые спектры УРЭ и ИВЭ для пленки с Cs при $n_{\min }$ остаточного О: $a$ - СУРЭ, $b-$ ПСИВЭ. $1-$ начальное состояние подложки; 2 - пленка с $\varphi_{\min }$ при адсорбции Cs; 3 - снятие экранирования цезием Ме участков подложки при прогреве пленки с $\varphi_{\min }, T=500-575 \mathrm{~K} ; 4-$ появление адсорбированного О на среднем уровне рельефа, $T=725 \mathrm{~K}$; 5 - переход О со среднего уровня на верхний и десорбция Cs из $\mathrm{W}_{2} \mathrm{OCs}$, NbCOCs и $\mathrm{NbW}_{2} \mathrm{OCs}, T=925 \mathrm{~K} ; 6-n_{\max } \mathrm{CO}$ после десорбции $\mathrm{Cs}, T=1075 \mathrm{~K}$. 
участков подложки адсорбатом без химических связей с нею [4]. Этот пик отвечает именно O, а не Cs, так как после начального уменьшения до $0.45 \mathrm{eV} E$ пика при $0.55 \mathrm{~V}$ она затем по достижении $\Delta \varphi=-1.5 \mathrm{eV}$ растет до $0.75 \mathrm{eV}$ и далее до $\Delta \varphi_{\min }$ не изменяется. Но $I$ этого пика по сравнению с начальной $I_{0}$ падает в $\sim 40$ раз. С учетом естественного соотношения выходов обратного упругого рассеяния электронов (УРЭ) для $\mathrm{Me}$ (а в особенности для пленок $\mathrm{Cs}^{+}$на Ме, склонных к захвату электронов зонда (т.е. к автоионизации)) и газовой пленки все это говорит о принадлежности пика при $0.75 \mathrm{~V} \mathrm{Cs}$, а не О. Поэтому $E=0.75 \mathrm{eV}$ отвечает уже поляризационной связи $\mathrm{Cs}^{+}-\mathrm{Me}$. Сильное экранирование подложки подтверждается еще сильным и синхронным с изменением $I$ пика с $E_{0}=0.55 \mathrm{eV}$ падением в 80 раз $I$ минимума при $1.15 \mathrm{~V}$, отвечающего $E$ возбуждения электронов подложки с $E_{0}=0.55 \mathrm{eV}$. Этот минимум по достижении $\varphi_{\min }$ уменьшает еще и $E$ до $0.9 \mathrm{eV}$ (рисунок, кривая 2,a). Значит, на последней стадии адсорбции Cs вытесняет оставшийся на верхнем уровне $\mathrm{O}$, не использованный им для химической связи или перемещения на более низкие уровни, в такие места подложки, где О не может образовывать даже молекул $\mathrm{W}_{2} \mathrm{O}$, т. е. в неупорядоченные дефекты верхнего уровня. О наличии там такого О говорит появление при $\Delta \varphi=-1.51 \mathrm{eV}$ ступеньки в СУРЭ при $1.9 \mathrm{~V}$ от поляризационной связи $\mathrm{O}$ с подложкой, точнее - от ее поляризованных $\delta$-связей Ме-Ме. Эта ступенька в ПСИВЭ не сдвигается (рисунок, кривая 2, $b$ ), что говорит об аморфности соответствующего адсорбата $[1,2]$. Подобное поведение пиков начальной области СУРЭ отмечается и в других системах Мe-O-Cs [13-16]. Интерпретация пика при $0 \mathrm{~V}$ во всех случаях одинакова, но второго - при $0.75 \mathrm{~V}$ в этой работе, $0.65 \mathrm{~V}$ для Мо [13] и $0.8 \mathrm{~V}$ для $\mathrm{Nb}$ [14] различна, что связано с существованием различных механизмов образования молекул суббронзы MeOCs $[9,13,14]$. В частности, полимеризация в пленке $\mathrm{Me}_{n} \mathrm{O}_{n} \mathrm{Cs}_{n}$ вызывает еще появление в СУРЭ пика при $\sim 7 \mathrm{~V}$ от $5 p$-плазмонов Cs [13].

Состояние пленки с пиком в СУРЭ при 0 V неустойчиво и разрушается при воздействии электронного пучка с $E_{p}>10 \mathrm{eV}$. Пик при этом теряет $I$ и смещается к $+0.2 \mathrm{~V}$, что говорит о том, что пленка становится островковой и, кроме того, состоит именно из физадсорбированного О. При $E_{p} \sim 25 \mathrm{eV}$ пик при $0 \mathrm{~V}$ исчезает сразу, а вместо него появляется при $0.35 \mathrm{~V}$, но исчезает сильный пик при $1.2 \mathrm{~V}$. Остальные же, за исключением пика от связи $\mathrm{Cs}^{+}-\mathrm{Me}$, повышающего $I$, не изменяются. Значит, при этой $E_{p}$ верхний уровень очищается настолько, что начинает „просвечивать“ подложка, хотя и слегка поляризованная оставшимся в островках $\mathrm{O}$, по которой из многослойного статистического покрытия может дополнительно „растекаться“ Cs.

Рост концентрации О на верхнем уровне в конце адсорбции Cs объясняется большим размером его иона $(0.33 \mathrm{~nm})$, из-за чего он не может проникать в некоторые объемные дефекты и поэтому разрывая слабую мостиковую связь в отдельных находящихся там молекулах $\mathrm{W}_{2} \mathrm{O}$ вынужден для образования донорно-акцепторной связи О-Cs извлекать О на более гладкую поверхность. Кроме того, в достаточно больших объемных дефектах в многослойном виде может присутствовать и не связанный со стенками О. На достаточно гладких и протяженных участках верхнего уровня Cs может легко образовать молекулы $\mathrm{W}_{2} \mathrm{OCs}$ с мостиковой связью $\mathrm{W}-\mathrm{O}-\mathrm{W}$ и донорно-акцепторной линейной (сильной) $\mathrm{O}-\mathrm{Cs}$, что в рассматриваемых дефектах затруднено изза невозможности образования для довольно большой молекулы оптимальной пространственной ориентации орбиталей W и О относительно орбиталей атомов подложки. Поэтому излишний для образования таких молекул О должен вытесняться в неупорядоченные дефекты верхнего уровня. А на менее протяженных гладких участках, например, ступеньках и террасах, остается возможной лишь адсорбция Cs на Ме. Такая пленка с учетом различий в $\varphi$ Cs и Мe может легко металлизоваться на общих с подложкой $s$-электронах. Поэтому появление при адсорбции Cs нового пика в СУРЭ при $1.35 \mathrm{~V}$ с большой вероятностью можно приписать поверхностным $6 s$-плазмонам $\mathrm{Cs}$, а небольшое уменьшение их $E$ относительно $1.55 \mathrm{eV}$ для массивного Cs или сплошной его пленки на Ме объяснить малыми размерами ее островков. Уменьшение концентрации О в объемных дефектах подтверждается еще и монотонным падением при адсорбции Cs $I$ пика при $15.7 \mathrm{~V}$, отвечающего несвязывающим $2 p$-орбиталям адсорбированного там О, вплоть до исчезновения этого пика вблизи $\varphi_{\min } . E$ этих орбиталей по сравнению с изолированным атомом О $(13.62 \mathrm{eV})$, естественно, должна быть увеличена из-за пониженного рельефа. Это понижение I вряд ли можно объяснить простым экранированием О цезием, так как описанный выше механизм извлечения О на поверхность энергетически более выгоден благодаря возможности образования сильной линейной химической связи O-Cs. Невозможность образования на подложке молекул $\mathrm{W}_{2} \mathrm{OCs}_{2}$ с привлечением для образования еще одной донорно-акцепторной связи O-Cs второй заполненной орбитали атомов О очевидна из-за большого размера иона $\mathrm{Cs}^{+}$.

Между молекулами $\mathrm{Nb}_{2} \mathrm{~W}_{2} \mathrm{O}$ на нижнем уровне, максимальная степень покрытия которых $\left(\Theta_{\max }\right)$ не может превышать 0.33 [9], в начальном состоянии подложки с большой вероятностью имеется физадсорбированный $\mathrm{O}$. Причем из-за упорядочения $\mathrm{Nb}_{2} \mathrm{~W}_{2} \mathrm{O}$ он принципиально не может там образовывать молекул WO или $\mathrm{W}_{2} \mathrm{O}[6,16,17]$. Кроме того, при наличии интерметаллического субоксида оксиды с материалом подложки на поверхности ни при каких условиях не образуются $[6,7]$. Это, по-видимому, и является основной причиной жаропрочности металлических сплавов. $\mathrm{Nb}_{2} \mathrm{~W}_{2} \mathrm{O}$ образуется на ПУ гранях или, в крайнем случае, ПУ рядах атомов подложки [16,17]. Естественно, что с большой вероятностью упорядочен и адсорбированный 
на нем О. В рассматриваемом случае $E_{0}$ пика при $2.35 \mathrm{~V}$ при адсорбции Cs изменяется мало - до $2.5 \mathrm{eV}$ при $\varphi_{\min }$. Его $I$ вплоть до $\Delta \varphi=-1.2 \mathrm{eV}$ не изменяется, до $\Delta \varphi=-1.5 \mathrm{eV}$ растет, но затем вплоть до получения $\varphi_{\min }$ не изменяется. С другой стороны, при адсорбции Cs монотонно падает $I$ пика при $10.8 \mathrm{~V}$, к получению $\varphi_{\min }$ уменьшаясь в $\sim 2.5$ раза. Значит, на неупорядоченных молекулах $\mathrm{W}_{2} \mathrm{O}$ с $E$ химической связи $\left(E_{\mathrm{b}}\right) \mathrm{W}-\mathrm{O}-\mathrm{W}$ $\sim 2.4 \mathrm{eV}$ Cs адсорбируется слабо, но зато хорошо на $\mathrm{O}$ между молекулами $\mathrm{Nb}_{2} \mathrm{~W}_{2} \mathrm{O}$ или же использует его для других процессов $(10.8 \mathrm{eV}$ - это $E$ несвязывающих $2 p$-орбиталей $\mathrm{O}$, уменьшенная с $13.62 \mathrm{eV}$ за счет его поляризации на гладких участках подложки).

Пик при $3.7 \mathrm{~V}$ от поверхностной связи $\mathrm{Nb}-\mathrm{O}$ в $\mathrm{Nb}_{2} \mathrm{~W}_{2} \mathrm{O}$ при адсорбции $\mathrm{Cs}$ до $\Delta \varphi=-1.1 \mathrm{eV}$ уменьшает $I$, далее до $\Delta \varphi=-1.43 \mathrm{eV}$ она стабилизируется, а затем немонотонно изменяясь восстанавливается до $I_{0}$. В то же время $I$ пика при $5.7 \mathrm{~V}$ от объемных связей $\mathrm{W}-\mathrm{O}-\mathrm{W}$ и $\mathrm{O}-\mathrm{Nb}$ в этом субоксиде к получению $\varphi_{\min }$ монотонно падает в $\sim 3$ раза. Так как исходя из большого размера иона $\mathrm{Cs}^{+}$трудно предположить экранирование в субоксиде одних только объемных связей, из этого следует, что Cs образует с ним химическую связь $\mathrm{O}-\mathrm{Cs}$, по $E_{\mathrm{b}}$ близкую к поверхностной $\mathrm{O}-\mathrm{Nb}[14,15]$. Образование связи Cs с субоксидом происходит за счет катализа, т. е. передачи пары электронов из поверхностной связи $\mathrm{Nb}-\mathrm{O}$ в связь $\mathrm{O}-\mathrm{Cs}$. При этом освободившиеся от связи с субоксидом атомы $\mathrm{Nb}$ смещаются в сторону от образующейся молекулы суббронзы $\mathrm{NbW}_{2} \mathrm{OCs}$ в соседнюю канавку между ПУ рядами атомов подложки по схеме, описанной в [9] для Cs на субоксиде $\mathrm{Nb}_{4} \mathrm{O}$. Очевидно, что ряды из таких атомов $\mathrm{Nb}$ (гребни) станут дополнительно создавать для молекул $\mathrm{NbW}_{2} \mathrm{OCs}$ пониженный потенциальный рельеф. Эти атомы $\mathrm{Nb}$ не могут растворяться в подложке из-за отсутствия общей с ее атомами $\mathrm{W}$ электронной системы [10] и наличия трехмерной решетки суббронзы с полностью насыщенными связями. Из-за неизменности в процессе адсорбции Cs $E_{0}$ и $I_{0}$ пика при $7.45 \mathrm{~V}$ от двойной связи $\mathrm{C}-\mathrm{O}$ в $\mathrm{W}_{2} \mathrm{CO}$, т.е. отсутствия ее экранирования цезием, трудно предположить образование связи Cs c верхним в этой молекуле $\mathrm{O}$ с той же $E$, что и у нижней $\mathrm{W}-\mathrm{C}-\mathrm{W}$. Очевидно, что мостиковый характер связи $\mathrm{W}-\mathrm{C}-\mathrm{W}$ и верхняя часть молекулы $\mathrm{W}_{2} \mathrm{CO}$ с двумя несвязывающими орбиталями О аналогичны тому же для $\mathrm{W}_{2} \mathrm{O}$. Но, как показано выше, в этих условиях Cs на $\mathrm{W}_{2} \mathrm{O}$ почти не адсорбируется. При образовании на поверхности сложных дипольных молекул, в особенности путем катализа, энергии крайних связей в них обычно стремятся к выравниванию $[14,15]$. Поэтому для $\mathrm{W}_{2} \mathrm{OCs}$ следовало бы ожидать $E_{\mathrm{b}} \mathrm{W}-\mathrm{C}-\mathrm{W}$ и $\mathrm{O}-\mathrm{Cs} \sim 2.5-3 \mathrm{eV}$. Однако при адсорбции Cs не совсем очевидно, что появляется новый пик с такой $E$, хотя $E_{0}$ пика при $2.35 \mathrm{~V}$ и смещается к $2.5 \mathrm{eV}$ почти без роста $I$. T. е. возможно, что слабая адсорбция $\mathrm{Cs}$ на $\mathrm{W}_{2} \mathrm{CO}$ так же, как и на $\mathrm{W}_{2} \mathrm{O}$, все же происходит. Трудность в интерпретации представляет не только близость $E_{\mathrm{b}} \mathrm{W}-\mathrm{O}-\mathrm{W}$ в $\mathrm{W}_{2} \mathrm{O}$ и $\mathrm{W}-\mathrm{C}-\mathrm{W}$ в $\mathrm{W}_{2} \mathrm{CO}$, но и почти неизменная $I$ пика с $E_{0}=2.35 \mathrm{eV}$, хотя обычное стягивание и уплотнение цезием под собой О путем полимеризации на связях $\mathrm{O}-\mathrm{O}$ или катализа при образовании молекул суббронзы $\mathrm{Me}_{n} \mathrm{O}_{n} \mathrm{Cs}_{n}$ должно бы приводить к росту $I$ этого пика.

Наконец, при попадании Cs в объемные дефекты, занятые $\mathrm{W}_{2} \mathrm{C}$, первый его сильно экранирует. Об этом говорит сильное падение (в 7 раз) I пика от связи $\mathrm{W}-\mathrm{C}-\mathrm{W}$ при $8.8 \mathrm{~V}$. Кроме того, это говорит и об отсутствии О в таких дефектах. В противном случае, подобно О в объемных дефектах без C, Cs извлекал бы на поверхность СО, чего в этих условиях в СУРЭ не наблюдается.

Описанной интерпретации процессов при адсорбции Cs помогает и рассмотрение ПСИВЭ, приведенных на рисунке (кривые $1, b$ и $2, b$ ), где по отсутствию энергетических сдвигов $\Delta E$ их пиков относительно СУРЭ (кривые $1, a$ и 2,a) или по их величине можно судить, находится ли соответствующий адсорбат в аморфном, слабо или достаточно хорошо упорядоченном состоянии [5].

\section{СУРЭ при прогреве пленок}

По окончании адсорбции Cs на не очищающемся полностью от примесей $\mathrm{Nb}, \mathrm{C}$ и $\mathrm{O}$ цилиндрическом газофазном поликристаллическом вольфрамовом покрытии сплава НбЦ-1 $(\mathrm{Nb}-1 \% \mathrm{Zr})$ с минимальной $n \mathrm{O}$, в частности, фиксируются пики: при $0 \mathrm{~V}$ в спектре упругорассеянных электронов (СУРЭ) [1,2], отвечающий полному экранированию подложки пленкой без химических связей, и при $1.35 \mathrm{~V}$ от поверхностных $6 s$-плазмонов $\mathrm{Cs}$. Они исчезают почти одновременно при температуре $T=550-575 \mathrm{~K}$ (см. рисунок, кривая 3). Это говорит о снятии экранирования некоторых участков верхнего уровня рельефа. Уже в самом начале прогрева пик при $0.75 \mathrm{~V}$ от связи $\mathrm{Cs}^{+}-\mathrm{Me}$ до $T=400 \mathrm{~K}$ уменьшает интенсивность $I$, особенно при $375 \mathrm{~K}$. Но это не означает десорбции Cs, так как полученная при адсорбции $\varphi$ не растет при прогреве, а падает. После достижения $\varphi_{\min }$ при $425 \mathrm{~K}$ даже начинается рост $I$ этого пика. Кроме того, два пика при 12.5 и $13.1 \mathrm{~V}$, которые к окончанию адсорбции Cs синхронно увеличивают $I$ в 1.7 раза и отвечают уже дублету от $5 p$-уровня $\mathrm{Cs}$, до $T=500 \mathrm{~K}$ сохраняют достигнутую $I$. Т. е. при таком прогреве происходит миграция Cs на другие химические состояния в островковых пленках более низких уровней.

Так, оказывается, что уже при 325-345 K наблюдается рост $I$ пика при $2.05 \mathrm{~V}$, отвечающего $\mathrm{W}_{2} \mathrm{O}$ без Cs. Но при $T=400 \mathrm{~K}$ его $I$ падает, при $T=450 \mathrm{~K}$ еще падает с уменьшением $E$ до $1.9 \mathrm{eV}$. Значит, Cs с Ме участков сюда не переходит, а напротив, с $\mathrm{W}_{2} \mathrm{O}$ снимается экранирование, а затем начинается его распад. Это и понятно, так как этот $\mathrm{W}_{2} \mathrm{O}$ при адсорбции Cs появляется только вблизи $\varphi_{\min }$. Т. е. его образование было стимулировано другими процессами, например, вытеснением лишнего 
для образования соединений c Cs остаточного O в поверхностные дефекты, где эти соединения образовываться не могут, с последующим его там уплотнением. Поэтому такая пленка вторичного $\mathrm{W}_{2} \mathrm{O}$ является слабо термоустойчивой и стоит только начаться миграции $\mathrm{Cs}$, как она станет диссоциировать. Действительно, I пика при $2.5 \mathrm{~V}$ от начального $\mathrm{W}_{2} \mathrm{O}$ до $550 \mathrm{~K}$ в общем не изменяется, а при $375 \mathrm{~K}$ даже возрастает. Т.е. с таким $\mathrm{W}_{2} \mathrm{O}$ Cs до этой $T$ не реагирует. На принадлежность пика с $E_{0}=3.65 \mathrm{eV}$ от поверхностной связи $\mathrm{Nb}-\mathrm{O}$ в $\mathrm{Nb}_{2} \mathrm{~W}_{2} \mathrm{O}$ в конце адсорбции Cs уже связи O-Cs в $\mathrm{NbW}_{2} \mathrm{OCs}$ указано выше. Действительно, уже при $325 \mathrm{~K}$ в СУРЭ появляется слабый пик при $3.95 \mathrm{~V}$, которого не было в течение всего процесса адсорбции Cs. До $450 \mathrm{~K}$ он не изменяется, но затем его $E$ падает до $3.8 \mathrm{eV}$, в то время как для пика с $E_{0}=3.65 \mathrm{eV}$ с $475 \mathrm{~K}$ начинает расти $E$ до тех пор пока при $575 \mathrm{~K}$ и $E=3.8 \mathrm{eV}$ оба пика не начинают совпадать (см. рисунок, кривая 3). Кроме того, при $325 \mathrm{~K}$ появляется еще один пик при $3.35 \mathrm{~V}$, который без изменения $I$ существует до $625 \mathrm{~K}$ и при $650 \mathrm{~K}$ исчезает. При $350 \mathrm{~K}$ появляется третий новый пик при $2.8 \mathrm{~V}$, который с плавным уменьшением $E$ до $2.5 \mathrm{eV}$ существует до $875 \mathrm{~K}$ и исчезает при $925 \mathrm{~K}$. В процессе адсорбции Cs все эти три пика не наблюдаются. Значит, при прогреве образуются как минимум три новых химических состояния. С учетом малых различий в $E_{\mathrm{b}} \mathrm{c}$ подложкой $\mathrm{W}_{2} \mathrm{CO}$ и $\mathrm{W}_{2} \mathrm{O}$ и необходимости расположения первого (и тем более возможного WCO) только на гладких участках, параллельных базовой поверхности, a второго, подходящего для связи с Cs - в желобах террас (в том числе и винтовых) или бороздках, пик при $2.8 \mathrm{~V}$ следует приписать образованию $\mathrm{W}_{2} \mathrm{OCs}$, а при $3.35 \mathrm{~V}-\mathrm{W}_{2}$ COCs.

Остается идентифицировать два пика - при 3.65 и $3.95 \mathrm{~V}$. Для этого обращается внимание на поведение пиков при $7.4 \mathrm{~V}$ от связи $\mathrm{C}-\mathrm{O}$ в $\mathrm{W}_{2} \mathrm{CO}$, при $8.65 \mathrm{~V}$ от связи $\mathrm{W}-\mathrm{C}-\mathrm{W}$ в $\mathrm{W}_{2} \mathrm{C}$ и при $10.75 \mathrm{~V}$ от $\mathrm{O}$ на $\mathrm{Nb}_{2} \mathrm{~W}_{2} \mathrm{O}$. На неизменность первого пика при адсорбции Cs указано выше. Однако уже вблизи $\varphi_{\min }$ его $I$ начинает расти. Этот рост продолжается и при прогреве до 325 и $375 \mathrm{~K}$. Но при $350,425,525,600-650 \mathrm{~K}$ его $I$ падает, не изменяясь при промежуточных $T$. I же пика при $8.75 \mathrm{~V}$ при этих $T$ по отношению к пику при $7.45 \mathrm{~V}$ изменяется в противофазе. Уже сама по себе немонотонность в изменении $I$ этих пиков исключает как экранирование, так и его снятие для соответствующих соединений. Значит, в объемных дефектах с $\mathrm{W}_{2} \mathrm{C}$ происходит какой-то химический процесс. Такое же изменение пика при $7.35 \mathrm{~V}$ в процессе прогрева до $650 \mathrm{~K}$ также не следует приписывать экранированию или снятию его и для $\mathrm{W}_{2} \mathrm{COCs}$. Так как на пленке $\mathrm{CO}$ на переходных Ме и их оксидах второй слой $\mathrm{Cs}^{+}$на гладких участках не образуется $[5,9,13-15,18,19]$, изменение $I$ указанных пиков следует трактовать, как изменение $n$ соответствующих адсорбатов. Исходя из результатов указанных работ можно полагать, что для данной подложки второй слой $\mathrm{Cs}^{+}$на пленках каких-либо соединений $\mathrm{O}$ c
Cs может адсорбироваться только на нижнем уровне. $\mathrm{O}$ наличии второго слоя $\mathrm{Cs}^{+}$на дипольной пленке $\mathrm{Me}-\mathrm{O}-\mathrm{Cs}$ всегда говорят: рост $\varphi$ при адсорбции Cs после получения $\varphi_{\min }$ вплоть до получения плато кривой $\varphi(t)$, т. е. наличие $\varphi_{p}$; уменьшение $\varphi_{p}$ в начальной стадии прогрева хотя бы до $\varphi_{\min }$; существование при прогреве эмиссии отрицательных ионов $\mathrm{Cs}^{-}$; а для монокристаллической подложки - еще и наличие соответствующей дифракционной картины от верхнего ГПУ слоя Cs c $\Theta_{\max }=0.33[13,18,19]$. В данном случае при прогреве наблюдаются слабое уменьшение $\Delta \varphi_{\min }$ на $0.05 \mathrm{eV}$ и эмиссия $\mathrm{Cs}^{-}$с плотностью тока $\sim 10^{-5} \mathrm{~A} \cdot \mathrm{cm}^{-2}$. Т. е. наличие небольшого количества $\mathrm{Cs}^{+}$на $\mathrm{NbW}_{2} \mathrm{OCs}$ все же не исключается. При достаточной $n \mathrm{Cs}^{+}$во втором слое на соединениях $\mathrm{Me}-\mathrm{O}-\mathrm{Cs}$ благодаря коллективизации внешних электронов за счет упорядочения пленки становится возможным возбуждение в этом слое поверхностных $5 p$-плазмонов Cs. При этом в СУРЭ обычно наблюдается пик при 6.7-7.2 V. Но в данном случае он еще не наблюдается.

I пика при $10.8 \mathrm{~V}$ от $\mathrm{O}$ на $\mathrm{Nb}_{2} \mathrm{~W}_{2} \mathrm{O}$ монотонно падает в 2.5 раза с адсорбцией $\mathrm{Cs}$, при прогреве до $325 \mathrm{~K}$ еще продолжает падать, затем в интервале 350-450 K не изменяется и лишь выше $475 \mathrm{~K}$ начинает расти. Значит, если трактовать рост $I$ как снятие экранирования $\mathrm{O}$ цезием, то оно может наступать лишь при $T>475 \mathrm{~K}$. Однако падение $I$ этого пика во время адсорбции Cs можно трактовать и как уменьшение $n$ этого О из-за того, что он может привлекаться цезием для других процессов химического взаимодействия.

\section{Структурные модели пленок}

С учетом описанных особенностей пиков в СУРЭ при $3.65,5.7,7.35,8.65$ и $10.8 \mathrm{~V}$ появление нового пика при $3.95 \mathrm{~V}$ и тот факт, что $\mathrm{Nb}_{2} \mathrm{~W}_{2} \mathrm{O}$ должен реагировать с Cs подобно $\mathrm{Nb}_{4} \mathrm{O}$ [13], для интерпретации отвечающих этим пикам процессов в начальной стадии прогрева пленки с $\varphi_{\min }$ можно предложить следующую модель. Часть ее для механизма образования суббронзы $\mathrm{NbW}_{2} \mathrm{OCs}$ уже описана выше. Поскольку $\Theta_{\max }$ молекул $\mathrm{NbW}_{2} \mathrm{OCs}$ не может из-за наличия $\mathrm{Nb}$ превышать 0.33 [13], они станут располагаться параллельными рядами вдоль ПУ рядов атомов подложки через три постоянных между ними, а освободившиеся в $\mathrm{Nb}_{2} \mathrm{~W}_{2} \mathrm{O}$ при катализе атомы $\mathrm{Nb}$ - между рядами молекул суббронзы. Образование в присутствии избыточного О этими атомами $\mathrm{Nb}$ молекул $\mathrm{NbO}$ на донорно-акцепторной связи $\mathrm{O}-\mathrm{Nb}$ или $\mathrm{Nb}_{2} \mathrm{O}$ на мостиковой $\mathrm{Nb}-\mathrm{O}-\mathrm{Nb}$ из-за тетраэдрической конфигурации внешних орбиталей О привело бы к появлению в этих молекулах соответственно одной или двух заполненных орбиталей $\mathrm{O}$ (не считая даже двух полузаполненных для $\mathrm{Nb}$ ), направленных к одному или обоим параллельным рядам $\mathrm{NbW}_{2} \mathrm{OCs}$, что вызвало бы их сильное отталкивание от Cs в суббронзе. Возможная еще для нее $\Theta=0.17$ в таких же, но наполовину 
разреженных, рядах [13] сути дела не изменит. Поэтому при образовании суббронзы существование $\mathrm{NbO}$ или $\mathrm{Nb}_{2} \mathrm{O}$ оказывается энергетически невыгодным. Эти же атомы $\mathrm{Nb}$, в отличие от подложки без $\mathrm{Cs}$, не могут образовывать и молекул типа $\mathrm{Me}_{3} \mathrm{O}$, т.е. $\mathrm{NbW}_{2} \mathrm{O}$ [15], так как требуемое для создания тетраэдрической конфигурации этой молекулы наличие атомов О во втором слое атомов подложки сдерживается решеткой уже существующей суббронзы, связывающей два слоя (верхний и второй) атомов подложки. Из структуры пленки $\mathrm{NbW}_{2} \mathrm{OCs}$ с учетом еще и наследуемой ею от субоксида $\mathrm{Nb}_{2} \mathrm{~W}_{2} \mathrm{O}$ релаксации атомов $\mathrm{Nb}$ и изгиба зон на границах ее островков с очевидностью следует ее низкая $\varphi$ и относительно высокая термоустойчивость. Максимальная термоустойчивость пленки с $\varphi_{\min }$ для монокристаллов обычно наблюдается для $\Theta_{\mathrm{Nb}}=0.17$ в случае $\mathrm{Nb}(110)[9,13,19]$ и приблизительно при такой же $\Theta_{\mathrm{Nb}}$ для сплава $\mathrm{Mo}-2.5 \% \mathrm{Nb}(110)[15,19]$.

Описанный процесс происходит в островках упорядоченной пленки, где $\Theta_{\mathrm{Nb}}$ не может превышать 0.33 , а $\Theta_{\mathrm{O}}$ в верхнем слое на $\mathrm{Nb}_{2} \mathrm{~W}_{2} \mathrm{O}-0.83[13,18]$. Отсутствие же на подложке ПУ граней или рядов атомов при образовании субоксида $\mathrm{Nb}_{2} \mathrm{~W}_{2} \mathrm{O}$ с неизбежностью должно привести к ее перестройке в ПУ, что уже само по себе понижает $E$ системы. Поэтому с учетом ненужности для $\mathrm{NbW}_{2} \mathrm{OCs}$ дополнительного верхнего $\mathrm{O}$ и невозможности образования с последним соединений вытесненными из субоксида атомами $\mathrm{Nb}$ излишний $\mathrm{O}$ при образовании $\mathrm{NbW}_{2} \mathrm{OCs}$ начнет вытесняться из пленки на границы островков или в дефекты.

Ион $\mathrm{Cs}^{+}$на поверхности может легко образовывать донорно-акцепторную связь с одной из двух заполненных орбиталей атома $\mathrm{O}$, не имеющей химической связи с подложкой. Отсутствие последней позволяет образовать оптимальную пространственную ориентацию на подложке молекулы монооксида CsO c оставшимися одной заполненной и двумя полузаполненными орбиталями атома О. Но такие молекулы $\mathrm{CsO}$, хотя и имеющие благодаря химической связи пониженную $E$ относительно несвязанных $\mathrm{O}$ и $\mathrm{Cs}$, тем не менее не будучи связанными с подложкой не понизят общую $E$ в смешанной пленке $\mathrm{NbW}_{2} \mathrm{OCs}+\mathrm{CsO}$. Поэтому оказывается более выгодным вытеснить $\mathrm{CsO}$ из этой пленки, в особенности, если существует возможность дальнейшего понижения $E$. Для этого нужно образовать с помощью двух заполненных орбиталей $\mathrm{O}$ в $\mathrm{CsO}$ двойную связь $\mathrm{O}-\mathrm{Cs}$ c молекулой $\mathrm{W}_{2} \mathrm{C}$ на стенке объемного дефекта, разрывая в ней связь $\mathrm{W}-\mathrm{C}-\mathrm{W}$, извлечь для создания оптимальной пространственной ориентации орбиталей комплекс COCs на более гладкие участки подложки, где нет субоксида $\mathrm{Nb}_{2} \mathrm{~W}_{2} \mathrm{O}$, например, на террасы более верхних уровней, и там опять образовать связь $\mathrm{W}-\mathrm{C}-\mathrm{W}$, т.е. в итоге молекулу $\mathrm{W}_{2} \mathrm{COCs}$. Таким образом, в присутствии Cs и дополнительного O может происходить стимулированное монооксидом $\mathrm{CsO}$ окисление карбида $\mathrm{W}_{2} \mathrm{C}$. Здесь приведен общий случай образования молекул типа $\mathrm{Me}_{2} \mathrm{COCs}$, когда у поверхностных атомов переходных
Ме нет вакантных $d$-орбиталей. Если же такие орбитали имеются, как, например, на участках с выходом ПУ атомов подложки, т.е. для ОЦК граней (110) и (112), или, как в рассматриваемом случае, всегда у освободившихся после образования $\mathrm{NbW}_{2} \mathrm{OCs}$ атомов $\mathrm{Nb}$ на нижнем уровне рельефа, то очевидно образование по тому же механизму соответственно WCOCs, а в последнем случае NbCOCs c более выгодными тройной связью $\mathrm{C}-\mathrm{O}$ и двумя донорно-акцепторными $\mathrm{C}-\mathrm{Nb}$ и $\mathrm{O}-\mathrm{Cs}$, т.е. с полностью насыщенными химическими связями. Естественно, что при наличии на среднем уровне выходов граней $\mathrm{W}(110)$ или (112) (Nb там отсутствует) и там возможно образование вертикально расположенных линейных молекул WCOCs. CO связывается с переходными Ме всегда только атомами С [5]. Очевидно, что $E$ тройной связи $\mathrm{C}-\mathrm{O}$ в NbCOCs, к тому же на нижнем уровне, должна быть больше $E$ двойной в $\mathrm{W}_{2} \mathrm{COCs}$ или даже тройной в WCOCs на среднем. Поэтому в полученной в начале прогрева пленке пик при $8.65 \mathrm{~V}$ теперь уже отвечает не связи $\mathrm{W}-\mathrm{C}-\mathrm{W}$ в $\mathrm{W}_{2} \mathrm{C}$, a тройной $\mathrm{C}-\mathrm{O}$ в NbCOCs. Интервал $E_{\mathrm{b}}$, в котором наблюдается этот пик без $\mathrm{Cs}$, составляет $8.2-9.15 \mathrm{eV}$, причем эта $E$ растет при $T>1875 \mathrm{~K}$. Значит, для $\mathrm{W}_{2} \mathrm{C}$ в более глубоких объемных дефектах $E_{\mathrm{b}} \mathrm{W}-\mathrm{C}-\mathrm{W}$ может быть и выше $E_{\mathrm{b}} \mathrm{C}-\mathrm{O}$ в $\mathrm{NbCO}$ или NbCOCs. Тогда становится понятным, что пик при $3.65 \mathrm{~V}$ отвечает $E_{\mathrm{b}}$ $\mathrm{O}-\mathrm{Cs}$ в NbCOCs, а при $3.95 \mathrm{~V}-E_{\mathrm{b}} \mathrm{O}-\mathrm{Cs}$ в $\mathrm{NbW}_{2} \mathrm{OCs}$. Последняя $E_{\mathrm{b}}$ выше первой из-за погруженности атомов $\mathrm{O}$ в $\mathrm{NbW}_{2} \mathrm{OCs}$ в подложку, где они связаны с нею тройной связью во втором слое ее атомов [13]. Поскольку в объемных дефектах может накапливаться больше $\mathrm{O}$, чем это необходимо для $\mathrm{NbCOCs} \mathrm{c} \Theta_{\max }=0.33$, излишние для его образования $\mathrm{O}$ и $\mathrm{COCs}$ станут вытесняться на верхние участки подложки, но для последних молекул обязательно на гладкие, например, основания террас среднего уровня. Так как $T$ полной десорбции с них СО на переходных Ме всегда выше $T$ полной десорбции $\mathrm{Cs}$, для загрязненной $\mathrm{C}$ подложки с $\mathrm{O}$ после полной десорбции Cs следует ожидать повышения на ней n CO относительно начальной.

Необходимость повышения $T$ для процессов образования $\mathrm{NbW}_{2} \mathrm{OCs}$ и NbCOCs связана как с малой $n_{0}$ $\mathrm{O}$ на $\mathrm{Nb}_{2} \mathrm{~W}_{2} \mathrm{O}$, так и с необходимостью увеличения его миграции для „поиска“ на сильно пересеченном рельефе молекул $\mathrm{W}_{2} \mathrm{C}$, расположенных явно не на тех участках, где имеются субоксид и адсорбированный на нем О. Миграция же Cs c более высоких уровней на нижний вызывается не только понижением там $E$ за счет рельефа и возможностью образования химических соединений, но и тем, что $n_{\max } \mathrm{Cs}$, который можно разместить на гладких участках подложки без химических связей $\left(\Theta_{\max }=0.33\right)[5,13,19]$, в два раза меньше требуемой для получения $\Theta_{\max }=0.67$ в смешанной пленке $\mathrm{NbW}_{2} \mathrm{OCs}+\mathrm{NbCOCs}$ с общей каркасной решеткой. Еще и поэтому описанные явления начинают наблюдаться только при прогреве. 
Так как процессы на нижнем уровне являются самыми выгодными, в случае недостатка на нем О для образования пленки $\mathrm{NbW}_{2} \mathrm{OCs}+\mathrm{NbCOCs}$ он может привлекаться и с более высоких. Действительно, начиная с $T=400 \mathrm{~K}$, происходит падение $I$ пика при $2.05 \mathrm{~V}$ от вторичного $\mathrm{W}_{2} \mathrm{O}$, а при $450 \mathrm{~K}$ она падает наиболее резко с уменьшением $E$ до $1.9 \mathrm{~V}$. T.е. диссоциирует даже часть молекул $\mathrm{W}_{2} \mathrm{O}$. I же пика при $3.65 \mathrm{~V}$ от связи O-Cs в $\mathrm{NbCOCs} \mathrm{при} \mathrm{этом} \mathrm{постоянно} \mathrm{растет.} \mathrm{С} \mathrm{другой} \mathrm{стороны,}$ начиная с $450 \mathrm{~K}$ растет I пика при $10.8 \mathrm{~V}$. Bсе это говорит о том, что на нижний уровень действительно поступает дополнительный О. Также очевидно, что для образования только $\mathrm{NbW}_{2} \mathrm{OCs}$ из $\mathrm{Nb}_{2} \mathrm{~W}_{2} \mathrm{O}$ не должно возникать проблем даже при отсутствии на подложке избыточного О.

Наблюдая за изменением $I$ и $E$ пика от связи $\mathrm{Cs}^{+}-\mathrm{Me}$ и учитывая, что их уменьшение означает десорбцию Cs c верхнего уровня, а рост $E$ без изменения или с ростом $I$ - переход Cs c верхнего на средний или нижний, можно установить, что при прогреве до $550 \mathrm{~K}$ часть Cs переходит на верхний уровень, а с $650 \mathrm{~K}$ оттуда уже начинается его десорбция. Это связано с избытком на подложке Cs для образования соединений на среднем и нижнем ее уровнях. В результате $n$ Cs на среднем уровне падает. Об этом говорит особенно сильный при $T=550 \mathrm{~K}$ рост $I$ следующего за пиком от этой связи минимума при $0.8-1.0 \mathrm{~V}$, отвечающего $E$ возбуждения электронов на чистых участках среднего уровня. Однако верхний при этом цезием полностью не покрывается. Об этом говорит сохранение АИМ при $0.15 \mathrm{~V}$ от почти чистых участков верхнего уровня, к тому же при неизменной $E$ (см. рисунок, кривая 3). Об образовании на среднем уровне $\mathrm{W}_{2} \mathrm{OCs}$ и $\mathrm{W}_{2} \mathrm{COCs}$ говорит появление отвечающих им пиков при 2.8 и $3.35 \mathrm{~V}$ от их $E_{\mathrm{b}}$ с подложкой только при прогреве пленки с Cs. Это, скорее всего, происходит в интервале $425-475 \mathrm{~K}$, так как при монотонном падении $I$ первого пика в СУРЭ при 0-0.1 V пик с $E_{0}=0.75 \mathrm{eV}$ в интервале $295-425 \mathrm{~K}$ уменьшает $I$, а в интервале $425-475 \mathrm{~K}$ - увеличивает. Поскольку при этом внешний поток Cs уже отсутствует, первый факт нельзя объяснить экранированием. Значит, в начале прогрева для образования химических соединений на среднем и нижнем уровнях может привлекаться Cs и с более высокого. Но начиная с $525 \mathrm{~K}$ не связанный в соединениях Cs уже вытесняется со среднего уровня на верхний. К более сильному уплотнению $\mathrm{Cs}$, чем с $\Theta_{\max }=0.33$ для ГПУ слоя $\mathrm{Cs}^{+}$на Ме, при взаимодействии его с О или СО может приводить только образование с помощью полимеризации или катализа дипольных молекул MeOCs или MeCOCs [5,9,13-15,18,19]. Значит, на основаниях террас среднего уровня, в том числе и винтовых, нет преобладания ПУ рядов атомов W и там наиболее вероятно образование именно молекул $\mathrm{W}_{2} \mathrm{OCs}$ и $\mathrm{W}_{2} \mathrm{COCs}$, требующих для этого как минимум в два раза меньше Cs, чем это необходимо для молекул WOCs или WCOCs. При выходе же ПУ рядов атомов W на наклонные стенки террас молекулы типа MeCOCs все равно не могут образовываться из-за необходимости их перпендикулярного расположения относительно базовой плоскости подложки.

Пик с $E_{0}=2.5 \mathrm{eV}$ от вторичного $\mathrm{W}_{2} \mathrm{O}$ при $T=450 \mathrm{~K}$ уменьшает $E$ и $I$, что говорит о диссоциации этого $\mathrm{W}_{2} \mathrm{O}$. Разуплотнившийся после этого О станет занимать уже бо́льшую площадь. Это также способствует вытеснению Cs на верхний уровень. Пик при $2.5 \mathrm{~V}$ соответствует первичному $\mathrm{W}_{2} \mathrm{O}$, поэтому уменьшение при $675 \mathrm{~K} E$ пика при $2.8 \mathrm{~V}$ до $2.5 \mathrm{eV}$ говорит о распаде при этой $T$ $\mathrm{W}_{2} \mathrm{OCs}$. Кроме того, почти одновременно с $\mathrm{W}_{2} \mathrm{OCs}$ происходит распад и $\mathrm{W}_{2} \mathrm{COCs}$. Отвечающий последнему пик при $3.35 \mathrm{~V}$ исчезает при почти той же $T(650 \mathrm{~K})$. При распаде на среднем уровне молекул $\mathrm{W}_{2} \mathrm{COCs}$ в них не обязательно должна разрываться именно связь O-Cs, в особенности на границах островков или террас, где связь с подложкой ослаблена. Так как террасы с большой вероятностью примыкают к совершенным участкам нижнего уровня с пленкой $\mathrm{NbW}_{2} \mathrm{OCs}+\mathrm{NbCOCs}$, причем последние молекулы в ней образуются за счет миграции COCs, в случае неоптимальной $n$ в смешанной пленке комплексы COCs c разрывом связей $\mathrm{W}-\mathrm{C}-\mathrm{W}$ в $\mathrm{W}_{2} \mathrm{COCs}$ могут с понижением $E$ переходить со среднего уровня на нижний, повышая на нем $n$ NbCOCs. $E$ при этом дополнительно падает еще и за счет образования сильных донорно-акцепторных связей $\mathrm{Nb}-\mathrm{C}$ и $\mathrm{O}-\mathrm{Cs}$ и перехода $\mathrm{CO}$ в молекуле NbCOCs к основному coстоянию с тройной связью $\mathrm{C}-\mathrm{O}$ [11]. Одновременное падение $I$ пиков при 3.4 и $7.4 \mathrm{~V}$ и рост ее для пиков при 3.65-3.8 и $8.7 \mathrm{~V}$ как раз и подтверждает указанный переход COCs. Очевидно, что такой переход энергетически выгоднее миграции COCs вдоль подложки через сильно пересеченный рельеф.

\section{Состояние подложки после десорбции цезия}

При $T=675 \mathrm{~K}$ между пиком при $0.3 \mathrm{~V}$ от связи $\mathrm{Cs}^{+}-\mathrm{Me}$ оставшегося на верхнем уровне Cs и минимумом при $0.9 \mathrm{~V}$, довольно значительная $E$ которого говорит о наличии чистых участков на среднем, появляются новые минимум при $0.4 \mathrm{~V}$ и максимум при $0.5 \mathrm{~V}$, при $725 \mathrm{~K}$ увеличивающие $E$ соответственно до 0.5 и $0.7 \mathrm{eV}$ и $I$ (см. рисунок, кривая 4). При $775 \mathrm{~K}$ их $I$ не изменяется, но при дальнейшем нагреве начинает падать вплоть до их исчезновения при $925 \mathrm{~K}$. Несколько ранее при $875 \mathrm{~K}$ исчезает минимум при $0.15 \mathrm{~V}$ и пик при $0.3 \mathrm{~V}$, что говорит о полной десорбции Cs c верхнего уровня. При $T=925 \mathrm{~K}$ исчезает пик с $E=0.7 \mathrm{eV}$, но появляется новый и сразу же очень сильный пик при $0.4 \mathrm{~V}$ (см. рисунок, кривая 5). Одновременно с ростом $E$ до $1.0 \mathrm{eV}$ резко растет $I$ минимума, а затем при $975 \mathrm{~K}$ еще раз так же резко. Кроме того, до $1.2 \mathrm{eV}$ растет и $E$ этого минимума. При дальнейшем прогреве новый пик с $E=0.4 \mathrm{eV}$ к $T=1125 \mathrm{~K}$ достигает $E=0.65 \mathrm{eV}$ и далее уже ни при какой $T$ не исчезает. Уже только из этого 
факта следует, что этот пик связан с неудаляемым О на верхнем уровне, точнее с плотностью поверхностных состояний $d$-электронов подложки, поляризованных адсорбированным в островках О. Поэтому существование в интервале $675-875 \mathrm{~K}$ пика при $0.5-0.7 \mathrm{~V}$ с соответствующим ему минимумом отвечает адсорбированному в островках О на среднем уровне. После десорбции Cs c верхнего уровня при $875 \mathrm{~K}$, приводящей к полной очистке последнего, этот О при $975 \mathrm{~K}$ резко переходит со среднего на верхний. Этот факт подтверждают сильный рост $I$ минимума при $1.0 \mathrm{~V}$, что говорит о резкой очистке среднего уровня, и исчезновение при $975 \mathrm{~K}$ пика от адсорбированного $\mathrm{O}$ с $E_{\mathrm{b}}=1.5 \mathrm{eV}$, до которой к этому моменту понижается в результате распада вторичного $\mathrm{W}_{2} \mathrm{O}$ его $E_{0}=2.05 \mathrm{eV}$. Следует также отметить, что именно при $675 \mathrm{~K}$ пик с $E_{0}=2.5 \mathrm{eV}$ от непрореагировавшего с Cs первичного $\mathrm{W}_{2} \mathrm{O}$ достигает $E_{\min }=2.2 \mathrm{eV}$. Значит, при прогреве пленки с Cs диссоциирует даже часть первичного $\mathrm{W}_{2} \mathrm{O} . T=925 \mathrm{~K}$ является для данной пленки критической. При ней одновременно происходят переход адсорбированного $\mathrm{O}$ со среднего уровня на верхний, полная десорбция $\mathrm{Cs}$ из $\mathrm{W}_{2} \mathrm{OCs}$, NbCOCs и $\mathrm{NbW}_{2} \mathrm{OCs}$. Полная же десорбция Cs c подложки происходит при $975 \mathrm{~K}$ и определяется его десорбцией из объемных дефектов с О. $T$ полной десорбции $\mathrm{Cs}$ из $\mathrm{W}_{2} \mathrm{OCs}$ определена по пику с $E=2.8 \mathrm{eV}$, когда он достигает $E=2.4 \mathrm{eV}$ и начиная с $T=975 \mathrm{~K}$ совпадает с пиком от первичного $\mathrm{W}_{2} \mathrm{O}$ без $\mathrm{Cs}$; из $\mathrm{NbCOCs}-$ по получению $I_{\max }$ пика при $8.65 \mathrm{~V}$, т.е. когда после десорбции из него Cs остается $\mathrm{NbCO}$ и снимается экранирование в нем связи С-О. Использование для интерпретации в последнем случае пика при $3.75 \mathrm{~V}$ от связи O-Cs проблематично, так как для данной пленки он совпадает с $E_{\mathrm{b}}$ поверхностной $\mathrm{O}-\mathrm{Nb}$ в одновременно восстанавливающемся $\mathrm{Nb}_{2} \mathrm{~W}_{2} \mathrm{O}$. Из $\mathrm{W}_{2} \mathrm{COCs} \mathrm{Cs}$, как указано выше, десорбируется еще при $650 \mathrm{~K} . T$ полной десорбции Cs из $\mathrm{NbW}_{2} \mathrm{OCs}$ определяется по $I_{\max }$ пиков при $\sim 4$ и $\sim 5.7 \mathrm{~V}$ от связи Ме-O в $\mathrm{Nb}_{2} \mathrm{~W}_{2} \mathrm{O}$. Обычно она близка к $T$ десорбции Cs из NbCOCs. Это и понятно, так как для восстановления $\mathrm{Nb}_{2} \mathrm{~W}_{2} \mathrm{O}$ требуются атомы $\mathrm{Nb}$ из NbCOCs. Полная десорбция Cs c Me участков определяется по исчезновению пика от связи $\mathrm{Cs}^{+}-\mathrm{Me}$. Полная десорбция Cs из объемных дефектов с О определяется по $I_{\max }$ пика при $\sim 15.5 \mathrm{~V}$ от заполненных $2 p$-орбиталей О. Полная же десорбция Cs c подложки вообще определяется по $I_{\min }$ дублета при 12.5 и 13.1 от $5 p$-уровня Cs. В зависимости от условий получения пленки, эта $I$ может совпадать с десорбцией Cs из какого-либо соединения. Кроме того, при этом резко возрастает $\varphi$.

Из приведенных примеров ясно, что многие процессы в полученной химически неоднородной пленочной системе прямо связаны друг с другом. Такая связь вызывается стремлением системы к $E_{\min }$. Но существуют процессы и с косвенной связью, например, образование вблизи $\varphi_{\min }$ вторичного $\mathrm{W}_{2} \mathrm{O}$, рост $n \mathrm{~W}_{2} \mathrm{O}$ при $T=375$, $525,550,875-925,1025-1075,1175,1275$ и $1575 \mathrm{~K}$ и ее уменьшение при $T=400,425,475,825,975,1125$, $1225,1325,1375$ и $1475 \mathrm{~K}$, немонотонное экранирование объемных дефектов - I соответствующего пика при $15.5-15.9 \mathrm{~V}$ растет при $T 550-650 \mathrm{~K}$ и $825-875 \mathrm{~K}$, а в остальных случаях вплоть до полной десорбции Cs при $975 \mathrm{~K}$ не изменяется.

Прогрев пленки после полной десорбции Cs показал следующее. При нагреве до $1025 \mathrm{~K}$ растет $I$ пика при $7.35 \mathrm{~V}$. I же пика при $8.7 \mathrm{~V}$ растет при $T=1075 \mathrm{~K}$ (см. рисунок, кривая 6). Рост последнего немонотонно продолжается до $1325 \mathrm{~K}$, в то время как $I$ первого остается постоянной. При $T=1375 \mathrm{~K}$ и выше $I$ обоих пиков уже падают. Это означает, что в присутствии на подложке Cs как на среднем, так и на нижнем уровнях растет содержание СО. Причины этого указаны выше. Так как выше $T=1375 \mathrm{~K}$ роста $I$ пика при $8.7 \mathrm{~V}$ уже не происходит, то это означает, что увеличения содержания С в заметных количествах в объемных дефектах за счет его диффузии из более глубоких слоев материала еще не происходит. Тогда рост $I$ этого пика при 1075, 1225 и $1325 \mathrm{~K}$ можно трактовать как рост n CO на нижнем уровне. Кроме того, рост n $\mathrm{CO}$ после десорбции $\mathrm{Cs}$ может быть связан еще и с дополнительным окислением части $\mathrm{W}_{2} \mathrm{C}$ физадсорбированным О. Этому способствует переход О в интервале $1175-1275 \mathrm{~K}$ с более высоких уровней на нижний. Об этом говорит падение $I$ пика при $0.65 \mathrm{~V}$. Хотя АИМ при $0 \mathrm{~V}$ появляется еще при $1025 \mathrm{~K}$ (см. рисунок, кривая 6) и сохраняется до $T=1425 \mathrm{~K}$, свидетельствуя о наличии на верхнем уровне чистых гладких участков, параллельных базовой поверхности, это говорит о том, что О переходит на нижний уровень именно из дефектов верхнего. При этом переходе падает $I$ дифракционного пика первого порядка от подложки при $13.1 \mathrm{~V}$ и растет $I$ пика от $\mathrm{O}$ на $\mathrm{Nb}_{2} \mathrm{~W}_{2} \mathrm{O}$. Часть этого $\mathrm{O}$ может попадать и в объемные дефекты, так как в этом же интервале $T$ растет и $I$ пика при $15.6 \mathrm{~V}$. Десорбция дополнительного СО с обоих уровней среднего и нижнего - происходит при $1375 \mathrm{~K}$. Однако слабый пик в СУРЭ при $7.4 \mathrm{~V}$ после $1375 \mathrm{~K}$ все же остается. Значит, полностью $\mathrm{CO}$ не удаляется, хотя в ЭОС при этом отсутствуют пики СО, но пики О, однако, остаются [9]. Значит, за счет увеличивающейся при этих $T$ диффузии С из объема все же происходит слабое окисление $\mathrm{W}_{2}$ С остаточным О. Чувствительность НЭСУРЭ к примесям как минимум на два порядка величины выше, чем в ЭОС [1,2]. В ЭОС в лучшем случае она не превышает 0.05 монослоя. При $T>1425 \mathrm{~K}$ начинается уже диффузия $\mathrm{O}$ в обратном направлении. Об этом говорят падение $I$ пиков при 10.8 и $15.6 \mathrm{~V}$ и рост $\varphi$ и $I$ пика при $0.65 \mathrm{~V}$. Последнее говорит о выходе О на верхний уровень. На нижнем происходит частичный распад $\mathrm{Nb}_{2} \mathrm{~W}_{2} \mathrm{O}$, скорее всего, на границах островков. Об этом говорит некоторое падение $I$ пика при $3.75 \mathrm{~V}$ от связей Ме-O в $\mathrm{Nb}_{2} \mathrm{~W}_{2} \mathrm{O}$ в интервале $T=1375-1875 \mathrm{~K}$. О появлении островков адсорбированного О именно на верхнем уровне также говорит и смещение АИМ от $0 \mathrm{~V}$ в область положительных $U$ на АТП $(+0.15 \mathrm{~V})$. 
Уменьшить же $n \mathrm{O}$ на верхнем уровне можно несколькими кратковременными вспышками (не более $1 \mathrm{~min}$ ) при более высоких $T$. При этом пики при 0.65 и $10.8 \mathrm{~V}$ уменьшают интенсивность, а при $3.75,5.6$ и $15.9 \mathrm{~V}$ ее увеличивают. Значит, при таком режиме прогрева несвязанный $\mathrm{O}$ десорбируется с верхнего и дополнительного нижнего уровней, но уже начинается выход $\mathrm{O}$ из более глубоких объемных дефектов с образованием дополнительных молекул $\mathrm{Nb}_{2} \mathrm{~W}_{2} \mathrm{O}$. Последнее связано, скорее всего, с постоянным улучшением совершенства поверхности при ее высокотемпературных прогревах (рекристаллизацией).

\section{Заключение}

Таким образом, при адсорбции Cs на начальном coстоянии подложки, т. е. при минимальной $n$ О, вначале уплотняется слабо связанный с нею О. Затем на этом $\mathrm{O}$ и также $\mathrm{O}$, расположенном между островками интерметаллического субоксида $\mathrm{Nb}_{2} \mathrm{~W}_{2} \mathrm{O}$ на нижнем уровне рельефа, происходит связывание О с Cs. Кроме того, Cs связывается и с молекулами $\mathrm{Nb}_{2} \mathrm{~W}_{2} \mathrm{O}$, образуя за счет катализа молекулы суббронзы $\mathrm{NbW}_{2} \mathrm{OCs}$. Одновременно на очищенных от О при его уплотнении участках подложки на поляризационной связи адсорбируется поступающий из потока $\mathrm{Cs}$, который, по достижении достаточной $n$ на менее дефектных (гладких) участках, металлизуется. Кроме того, Cs может извлекать на поверхность О из некоторых объемных дефектов, не содержащих $\mathrm{C}$ или $\mathrm{W}_{2} \mathrm{C}$. Молекулы же $\mathrm{W}_{2} \mathrm{C}$ в других объемных дефектах экранируются цезием без химических связей. На небольшом количестве остаточного СО Cs практически не адсорбируется. Вытесняя лишний кислород с мест, где химическая связь с Cs образуется, в места, где она невозможна, Сs стимулирует там образование вторичных молекул $\mathrm{W}_{2} \mathrm{O}$. Оно из-за уплотнения $\mathrm{O}$, скорее всего, происходит в линейных дефектах типа канавок, бороздок и желобов террас.

При прогреве пленки с $\varphi_{\min }$ происходят следующие процессы. Из-за малой $n \mathrm{O}$, неравномерного распределения адсорбатов, связанного с их затрудненной миграцией при $295 \mathrm{~K}$ по сильно пересеченному рельефу, при низких $T$ в основном происходит перераспределение Cs и химически не связанного с подложкой О. Оно приводит к их миграции в виде химически не связанных с подложкой молекул $\mathrm{CsO}$ с верхнего уровня на нижние с образованием на среднем соединений $\mathrm{W}_{2} \mathrm{OCs}$ в желобах террас и $\mathrm{W}_{2} \mathrm{COCs}$ на их основаниях, а на нижнем - смешанной пленки $\mathrm{NbW}_{2} \mathrm{OCs}+\mathrm{NbCOCs} \mathrm{c}$ каркасной структурой. При этом излишний для образования соединений c Cs O вытесняется в линейные дефекты, где возможно образование вторичных молекул $\mathrm{W}_{2} \mathrm{O}$. Образование суббронзы $\mathrm{NbW}_{2} \mathrm{OCs}$ на нижнем уровне происходит за счет катализа. При этом от связи с $\mathrm{O}$ в $\mathrm{Nb}_{2} \mathrm{~W}_{2} \mathrm{O}$, связывающем два верхних слоя атомов подложки, освобождаются верхние атомы $\mathrm{Nb}$ и, передавая электроны этой связи цезию, смещаются в сторону. Образование $\mathrm{W}_{2} \mathrm{COCs}$ на среднем и $\mathrm{NbCOCs}$ на нижнем уровнях даже при отсутствии на подложке остаточного СО происходит за счет стимулированного молекулами $\mathrm{CsO}$ окисления карбида $\mathrm{W}_{2} \mathrm{C}$ в объемных дефектах с последующей миграцией COCs на гладкие участки нижнего и среднего уровней. Миграции COCs на террасы среднего уровня способствуют еще и ограничение по $\Theta_{\max }$ для свободных атомов $\mathrm{Nb}$ на нижнем. При этом верхний уровень способен полностью очищаться от адсорбатов. При прогреве в интервале 425-650 K на среднем уровне рельефа происходит постепенный (из-за различного размера островков) распад $\mathrm{W}_{2} \mathrm{OCs}, \mathrm{W}_{2} \mathrm{COCs}$ и вторичного $\mathrm{W}_{2} \mathrm{O}$, повышающий там $n \mathrm{Cs}$, не связанного в соединениях с подложкой, и частично О. В результате из-за ограничений по площади для разуплотняющихся адсорбатов на террасах $\mathrm{Cs}$, удерживаемый подложкой слабее, чем О, выше $525 \mathrm{~K}$ начинает вытесняться на свободный от адсорбатов верхний уровень. При $600 \mathrm{~K}$ начинается десорбция Cs c этого уровня, наиболее сильная при $650 \mathrm{~K}$. Полная же десорбция Cs c этого уровня происходит при $875 \mathrm{~K}$ и связана с постоянным поступлением на него Cs при распаде соединений на нижнем. И сразу же после десорбции Cs туда начинает поступать О со среднего уровня. Но после полной десорбции Cs при $975 \mathrm{~K}$ из объемных дефектов с О часть этого О поступает и на нижний, наиболее сильно после прогрева при 1175-1275 К. Кроме того, после распада $\mathrm{W}_{2} \mathrm{COCs}$, в особенности на границах террас с нижним уровнем рельефа, часть COCs с понижением $E$ может переходить на него со среднего, оптимизируя $n$ молекул $\mathrm{NbCOCs}$ в смешанной пленке $\mathrm{NbW}_{2} \mathrm{OCs}+\mathrm{NbCOCs}$, в особенности если для их образования не хватало О даже при достаточной $n$ в дефектах С. О выгодности этого процесса свидетельствует, в конце концов, полный переход COCs co среднего уровня на нижний при 650 K. После полной десорбции Cs на подложке остается повышенная относительно начальной концентрация СO, в основном на нижнем уровне. Его присутствие там объясняется десорбцией Cs из $\mathrm{NbW}_{2} \mathrm{OCs}$ и NbCOCs, происходящей почти одновременно из-за того, что при восстановлении $\mathrm{Nb}_{2} \mathrm{~W}_{2} \mathrm{O}$ привлекаются атомы $\mathrm{Nb}$ из распадающегося NbCOCs. Поэтому после десорбции $\mathrm{Cs}$ из последнего разрушается и субкарбонил $\mathrm{NbCO}$. Десорбция освободившегося в результате этого СО и также $\mathrm{CO}$, дополнительно образовавшегося в интервале $1075-1325 \mathrm{~K}$ за счет окисления части $\mathrm{W}_{2} \mathrm{C}$ или С остаточным О, происходит при 1375 К. При 1425 К начинается выход О на более высокие уровни из объемных дефектов нижнего. Уменьшения его $n$ на верхнем уровне можно добиться несколькими вспышками при $T \geq 1875 \mathrm{~K}$. При этом за счет рекристаллизации материала образца и диффузии из подложки $\mathrm{Nb}$ для $\mathrm{W}$ покрытия на нижнем уровне несколько увеличивается и $n \mathrm{Nb}_{2} \mathrm{~W}_{2} \mathrm{O}$. Таким образом, последствия присутствия Cs на начальном состоянии подложки сводятся к минимуму при $1375 \mathrm{~K}$, а без учета образования дополнительного СО - при 
$975 \mathrm{~K}$. Полученное при $425 \mathrm{~K}$ в этой части работы $\Delta \varphi_{\min }=-1.68 \mathrm{eV}$, если для поликристаллического $\mathrm{W}$ принять рекомендованное в [20] значение $\varphi=4.45 \mathrm{eV}$, с учетом начального (без $\mathrm{Cs}$ ) $\Delta \varphi_{0}=-0.25 \mathrm{eV}$, приводит к $\varphi_{\min }=2.52 \mathrm{eV}$ что на $\sim 1.4 \mathrm{eV}$ выше получаемой для оптимальной пленочной системы $\mathrm{Me}-\mathrm{O}-\mathrm{Cs}$ на монокристаллах $[5,9,10,13-16,18,19]$. Перемещая зондирующий пучок электронов по поверхности образца, можно найти на нем участки и с $\Delta \varphi_{0}=-0.42 \mathrm{eV}$ [7], т.е. получить с Cs $\varphi_{\min }=2.35 \mathrm{eV}$. Связана ли эта повышенная по сравнению с системой $\mathrm{W}(110)-\mathrm{O}-\mathrm{Cs}[10,16] \varphi_{\min } \mathrm{c}$ недостаточной $n$ на подложке $\mathrm{O}$ или наличием примесей $\mathrm{C}, \mathrm{W}_{2} \mathrm{C}$ и в особенности $\mathrm{CO}$, предстоит выяснить в последующей работе с пленками $\mathrm{Me}-\mathrm{O}-\mathrm{Cs}$ на этой подложке.

\section{Конфликт интересов}

Авторы заявляют, что у них нет конфликта интересов.

\section{Список литературы}

[1] Зыков Б.М., Нардая Ю.И., Сабельников А.М. // РХЖ. 2003. Т. 47. Вып. 2. С. $70-80$.

[2] Зыков Б.М., Нардая Ю.И. // Поверхность. 2003. № 8. C. $61-73$.

[3] Зыков Б.М., Сабельников А.М., Цхакая В.К. // Поверхность. 1987. № 9. С. 76-83.

[4] Зыков Б.М., Сабельников А.М. // Высокочистые вещества. 1990. № 5. C.123-129.

[5] Зыков Б.М., Кобяков В.П., Нардая Ю.И. // Высокочистые вещества. 1991. № 1. С. $71-80$.

[6] Бравареи А.В., Зыков Б.М., Зыкова В.Н., Лебедев В.Н., Удовиченко Ю.К. // ЖТФ. 2002. Т. 72. Вып. 9. С. 112-118.

[7] Зыков Б.М., Красненкова Т.М., Лазба Б.А. // ЖТФ. 2019. Т. 89. Вып. 8. С. $1300-1308$.

[8] Зыков Б.М., Цхакая В.К. // ЖТФ. 1979. Т. 49. Вып. 8. C. $1700-1707$.

[9] Зыков Б.М., Сабельников А.М. // Поверхность. 1988. № 10. C. $61-70$.

[10] Зыков Б.М., Кобяков В.П., Нардая Ю.И. // Высокочистые вещества. 1991. № 4. С. 125-136.

[11] Семенов И.Н., Овчинников К.В. „Неожиданные“ неорганические соединения. Л.: Химия, 1972. 105 с.

[12] Зыков Б.М., Цхакая В.К., Ярыгин В.И. // Открытия и изобретения. 1983. № 7. С. 236. А.С. № 1062803.

[13] Зыков Б.М., Сабельников А.М., Цхакая В.К. // Поверхность. 1990. № 6. С. 48-55.

[14] Зыков Б.М. Сабельников А.М. // Поверхность. 1990. № 9. C. $22-29$.

[15] Зыков Б.М., Нардая Ю.И., Сабельников А.М. // Высокочистые вещества. 1991. № 4. С. 116-124.

[16] Зыков Б.М., Нардая Ю.И. // ЖТФ. 1995. Т. 65. Вып. 4. C. $150-166$.

[17] Зыков Б.М., Иконников Д.С., Цхакая В.К. // ФТТ. 1975. Т. 17. Вып. 12. С. 3562-3567.

[18] Зыков Б.М., Цхакая В.К. // ЖТФ. 1980. Т. 50. Вып. 8. C. $1771-1780$.

[19] Зыков Б.М., Сабельников А.М., Цхакал В.К. // Поверхность. 1986. № 12. С. 21-29.

[20] Фоменко В.С. Эмиссионные свойства материалов. Киев: Наукова думка, 1981. 340 с. 